\title{
Estructuralismo histórico, economía política y teorías de la comunicación: notas sobre la trayectoria del pensamiento crítico latinoamericano
}

\section{Mauricio Herrera-Jaramillo ${ }^{1}$ César Bolaño²}

Recibido: 22/02/2018

Aprobado por pares: 30/03/2018
Enviado a pares: 23/02/2018

Aceptado: 30/04/2018

DOI: 10.5294/pacla.2019.22.2.7

Para citar este artículo / to reference this article / para citar este artigo Herrera-Jaramillo, M. y Bolaño, C. (2019). Estructuralismo histórico, economía política y teorías de la comunicación. Notas sobre la trayectoria del pensamiento crítico latinoamericano. Palabra Clave, 22(2), e2227. DOI: 10.5294/pacla.2019.22.2.7

\section{Resumen}

Este trabajo identifica la primera escuela crítica en comunicación y el estructuralismo latinoamericano como bases teóricas de la economía política de la comunicación (EPC) y de la cultura brasileña. Basados en la trayectoria de Luis Ramiro Beltrán, y de manera específica en su trabajo La revolución verde y el desarrollo rural latinoamericano, en que trabaja la categoría de efecto de demostración, son analizadas las limitantes teóricas de esta escuela una vez identifica la naturaleza estructural del subdesarrollo en América Latina. Así, partiendo de la necesidad de evidenciar la doble contradicción del capital (capital/trabajo y economía/cultura), la EPC pone el problema del papel de los medios de comunicación de masas en el plano de las mediaciones sociales, basados en la apropiación por parte del capital de los elementos de la cultura popular que, pasando primero por un momento inicial de acumulación primitiva de conocimiento, constituirá posteriormen-

1 orcid.org/0000-0001-5740-547X. Universidad de São Paulo, Brasil. mauricio.herreraj@usp.br

2 orcid.org/0000-0001-5756-7049. Universidade Federal de Sergipe, Brasil. bolano@ufs.br 
te la cultura industrializada. Emerge, entonces, la industria cultural como la instancia de mediación característica del capitalismo monopolista, y es precisamente allí, apoyado en la teoría de la dependencia cultural de Celso Furtado, desde donde la EPC brasileña supera la limitante ya identificada por Ingrid Sarti, al plantear que, bajo la idea de cultura de masas, se encubre la existencia de una cultura de clase.

\section{Palabras clave (fuente: tesauro de la Unesco)}

Pensamiento crítico; dependencia cultural; economía política de la comunicación; economía política de la comunicación y de la cultura; medios de comunicación de masas; Luis Ramiro Beltrán; Brasil; Celso Furtado; Ingrid Sarti; estructuralismo histórico. 


\section{Historical Structuralism, Political Economy and Communication Theories: Notes on the Development of Latin American Critical Thinking}

\section{Abstract}

This paper identifies the first school of communication criticism and Latin American structuralism as theoretical bases of the political economy of communications (PEC) and Brazilian culture. In reference to the career of Luis Ramiro Beltrán, specifically his work $L$ a revolución verde y el desarrollo rural latinoamericano - in which he discusses the category of demonstration effect-, the theoretical limitations of this school are analyzed after identifying the structural nature of underdevelopment in Latin America. Thus, starting from the need to show the double contradiction of capital (capital/labor and economy/culture), the PEC raises the problem of the role of mass media at the level of social mediations, based on capital's adoption of popular culture elements that - passing first through a moment of primitive knowledge accumulation - will later constitute the industrialized culture. Then, the cultural industry emerges as the mediation authority that characterizes monopoly capitalism, and this is where, supported by Celso Furtado's theory of cultural dependence, the Brazilian PEC exceeds the constraint already recognized by Ingrid Sarti when she stated that the idea of mass culture conceals the existence of a class culture.

\section{Keywords (source: Unesco Thesaurus)}

Critical thinking; cultural dependence; political economy of communication; political economy of communication and culture; mass media; Luis Ramiro Beltrán; Brazil; Celso Furtado; Ingrid Sarti; historical structuralism. 


\section{Estruturalismo histórico, economia política e teorias da comunicação: notas sobre a trajetória do pensamento crítico latino-americano}

\section{Resumo}

Este trabalho identifica a primeira escola crítica de comunicação e o estruturalismo latino-americano como bases teóricas da economia política da comunicação (EPC) e da cultura brasileira. Com base na trajetória de Luis Ramiro Beltran e, especificamente, em sua obra A Revolução Verde e o Desenvolvimento Rural da América Latina, na qual trabalha a categoria de efeito de demonstração, são analisadas as limitações teóricas dessa escola, já que se identifica a natureza estrutural do subdesenvolvimento na América Latina. Sendo assim, a partir da necessidade de destacar a dupla contradição do capital (capital/trabalho e economia/cultura), a EPC coloca o problema do papel dos meios de comunicação de massa no âmbito das mediações sociais, com base na apropriação que o capital faz de elementos da cultura popular que, primeiramente, passando por um momento inicial de acumulação primitiva de conhecimento, constituirá posteriormente a cultura industrializada. Emerge, então, a indústria cultural como a instância de mediação característica do capitalismo monopolista e é exatamente nela, com base na teoria da dependência cultural de Celso Furtado, que a EPC brasileira excede a limitante já identificada por Ingrid Sarti, que propõe que sob a perspectiva da cultura de massas encobre-se a existência de uma cultura de classe.

\section{Palavras-chave (fonte: tesauro da Unesco)}

Pensamento critico; dependência cultural; economia política da comunicação; economia política da comunicação e cultura; meios de comunicação social; Luis Ramiro Beltrán; Brasil; Celso Furtado; Ingrid Sarti; estruturalismo histórico. 


\section{Introducción}

Este artículo parte de la idea de que, a diferencia de la proletarización vía expansión de la relación salarial que se dio en el campo latinoamericano con la revolución tecnológica que representaron los monocultivos de maíz, arroz, trigo, entre otros, donde la subordinación al capital podía expresarse bajo la fórmula marxiana de la transición de la subsunción formal a la real, en las comunidades rurales, la subordinación se produjo bajo los moldes de lo que proponemos llamar "subsunción por imposición de conocimiento".

Recurriendo al caso del pequeño propietario de tierra que produce tabaco en el estado brasileño de Santa Catalina, quien sometido al poder del monopolio-monopsonio de la gran industria tabacalera internacional debía comprar todos los insumos a aquella industria, incluso recurriendo a crédito ofrecido por ella misma o sus filiales comerciales o financieras, y además tenía que comprometer la venta de todo su producto a ella, eximiéndose, aquella empresa, de toda la responsabilidad que implicaría mantener un cuadro de trabajadores rurales asalariados, planteamos que no existe entonces, en ese caso, subsunción formal y, por ende, tampoco se puede hablar de subsunción real del trabajo en el capital. Sin embargo, sí existe claramente una forma muy desarrollada de subsunción material, en la medida en que el proceso concreto de trabajo queda completamente bajo el control efectivo del referido gran capital.

Esta nueva forma de control se establece gracias a la superioridad científica y tecnológica norteamericana, desarrollada, principalmente, a partir del siglo pasado bajo la difusión del modelo de la revolución verde, que representa una nueva forma de acumulación primitiva de capital que, reproduciendo las condiciones de trabajo libre y permitiendo que el campesino acceda a la tierra, encubre el carácter explotador del capital. Pues, en la medida en que integra el trabajador "independiente" a la lógica del mercado (del consumo), atomizando y fragmentando las comunidades y con ello los valores y principios asociados a sus modos de vida tradicionales, crea materialmente las condiciones objetivas para el control del trabajo. 
En cuanto que ese análisis muestra la importancia de variables extraeconómicas a la hora de analizar la relación desarrollo-subdesarrollo, tema clave en las discusiones principalmente económicas de las décadas de los sesenta y setenta, es recuperada la discusión en torno a la relación cultura-desarrollo traída por Celso Furtado y, en general, por el estructuralismo latinoamericano, la cual permite redimensionar el problema del subdesarrollo, al entenderlo, principalmente, como un proceso, primero, de histórica dependencia cultural y, después, en el momento de la industrialización periférica, de dependencia tecnológica, la cual se materializa como un proceso de enraizamiento en la estructura productiva de aquella dependencia cultural, pues los avances de la técnica se dan jalonados por las necesidades de satisfacer la diversificación de los patrones de consumo de los grupos privilegiados de las periferias.

La base del proyecto modernizador norteamericano de superioridad científica fue la generación de una nueva estructura de relaciones internacionales con América Latina, sustentada en una doble estrategia de difusión científica y penetración cultural. Para ello, tres elementos fueron claves: ciencia, educación y comunicación, los cuales, más que procesos aislados, se materializaron de forma simultánea basados en una infraestructura institucional común y particular (en torno a la Organización de las Naciones Unidas [ONU]).

Con el propósito de aportar al elemento de comunicación de esta estrategia, este trabajo propone el diálogo con al menos tres importantes bibliografías: el estructuralismo histórico latinoamericano y las teorías del desarrollo, las teorías de la dependencia o del imperialismo cultural, derivadas de las teorías de la dependencia y que a la postre forman la primera escuela crítica del pensamiento comunicacional latinoamericano, y la llamada economía política de la comunicación y de la cultura, que encuentra también en América Latina particularidades que la diferencian de otras escuelas, europeas y norteamericanas de enfoque similar. Dados los límites de extensión de este artículo, no desarrollamos un diálogo amplio entre estas y otras corrientes importantes de pensamiento como la de los estudios culturales, también referenciado en el texto; sin embargo, consideramos 
que puede contribuir también a esta escuela al profundizar muy específicamente en una categoría transversal a las tres corrientes planteadas: el efecto de demostración. La estrategia argumentativa que adoptamos fue la de poner en diálogo dos de los más importantes intelectuales vinculados a las dos primeras escuelas citadas, Celso Furtado y Luis Ramiro Beltrán, respectivamente, con la intención de presentar la forma de apropiación de esta categoría por la vertiente crítica de la economía política de la comunicación y de la cultura (EPC), a la cual los autores de este trabajo pertenecen. Recuperar este viejo concepto tiene por objetivo proponer un diálogo necesario para el avance del pensamiento crítico latinoamericano en un momento de cambios fundamentales que afectan los diferentes campos intelectuales, en particular el de la comunicación. Por tanto, la contribución es innovadora, no solo respecto de su contenido específico, sino especialmente por constituirse en una propuesta de diálogo inédita, basada en una metodología poco utilizada de revisitar los clásicos para promover el diálogo entre contribuciones aparentemente distantes, según una categoría focal, a partir de la cual otros desarrollos, otros diálogos, pueden ser realizados, explorando una particular frontera de conocimiento.

En un primer momento, y con el objetivo de contextualizar el marco internacional en el que surge la comunicación para el desarrollo en América Latina, uno de los mecanismos utilizados para la ejecución de la doble estrategia antes mencionada, presentamos de manera sintética su contenido destacando su materialización a través de la subsunción por imposición de conocimiento. A continuación, se da cuenta del contexto en que surge la primera escuela de pensamiento crítico latinoamericana en comunicación (dependencia cultural o imperialismo cultural), para entonces evidenciar la crítica hecha a finales de la década de los setenta por Ingrid Sarti respecto de que, bajo la idea de cultura de masas, resaltada desde un análisis de relaciones entre naciones, se encubre la existencia de una cultura de clase y su contradicción inherente. Puesta esta crítica, recuperamos el concepto de efecto de demostración a partir de los aportes de dos grandes representantes de las escuelas puestas en discusión, con el objetivo de mostrar las potencialidades y limitantes del concepto para explicar la profundización 
del subdesarrollo y la dependencia en los países de América Latina por la vía de la modernización de los patrones de consumo. Finalmente, si en las teorías del imperialismo cultural el problema de la dependencia, como veremos, era entendido como un proceso de imposición cultural a través de los medios masivos, desde la perspectiva de la EPC, tal como aquí se defiende, articulada con el estructuralismo latinoamericano de Furtado, las industrias culturales deben ser entendidas como una instancia de mediación que desempeña un doble papel, pues, por un lado, favorece la integración internacional de los países periféricos, pero, por otro, de manera contradictoria, incentiva procesos de desintegración interna en favor de grupos privilegiados locales. Así, entonces, la EPC pone el problema del papel de los medios de comunicación de masas en el plano de las mediaciones sociales, basados en la apropiación por parte del capital de los elementos de la cultura popular que, pasando primero por un momento inicial de acumulación primitiva de conocimiento (Bolaño, 2013), constituirá posteriormente la cultura industrializada. Emerge, entonces, la industria cultural como la instancia de mediación característica del capitalismo monopolista, y es precisamente allí donde, apoyada en la teoría de la dependencia cultural en Furtado, la EPC brasileña propone una alternativa para superar la limitante identificada por Sartri y el concepto de educación para el desarrollo (ED) propuesto históricamente para entender el subdesarrollo fuera del sistema de producción.

Así entonces, la reproducción de la dependencia cultural implica modificación de modos de consumo y de vida en consonancia con los cambios en las relaciones sociales de producción, en que la dependencia tecnológica también se reproduce, modificando las formas de subsunción del trabajo y los procesos productivos. Como diría Marx, una vez concluido, el proceso no deja huellas. Entenderlo exige aclarar su génesis y las diferentes estrategias a través de las cuales el capital se ha apropiado de las culturas tradicionales, dejando en manos de las industrias culturales ( $y$ otras instancias de mediación) el papel de mediación social por subsunción del trabajo cultural en el capital. 


\section{Difusión científica y penetración cultural}

\section{Hegemonía de los Estados Unidos y el pensamiento crítico latinoamericano}

El punto de partida de la propuesta que desarrollamos es la reformulación de la política de relaciones internacionales para América Latina que los Estados Unidos estructuran a partir de la década de los treinta con el objetivo de alinear a América Latina de su lado como estrategia política y económica ante la guerra. Así, iniciando con la Política de Buena Vecindad del presidente Franklin D. Roosevelt en la década de los treinta, posteriormente el Punto IV de Harry Truman en 1948 y finalmente la Alianza para el Progreso de John F. Kennedy, se estructuraría una estrategia de relaciones internacionales basada en dos pilares fundamentales: por un lado, la difusión científica, y por el otro, la penetración cultural.

Las bases de esta estrategia son resultado de décadas de trabajo norteamericano en investigación y experimentación en germoplasma, cultivo y evaluación de semillas modificadas genéticamente y diversos tipos de programas de naturaleza "social" (extensión rural, crédito, alfabetización, etc.), bajo el liderazgo de Nelson Rockefeller, quien promovió y orquestó una serie de alianzas entre capital privado y público norteamericano con las élites locales y los gobiernos de los países latinoamericanos a través de toda una infraestructura institucional complementaria a la multilateral (Banco Mun$\operatorname{dial}[\mathrm{BM}]$, Fondo Monetario Internacional [FMI], etc.), que garantizaron, vía política pública y de cooperación internacional, esa doble estrategia fundada en la superioridad científica y tecnológica, y en el mito del desarrollo.

Si bien todos los desarrollos tecnológicos propios de la carrera armamentista de la Segunda Guerra Mundial fueron fundamentales para estos avances, serían proyectos como el Centro Internacional del Mejoramiento de Maíz y Trigo (CIMMYT) los que posteriormente abrirían las fronteras "agriculturales" nacionales e internacionales para la circulación de semillas y variedades entre regiones y países, "consolidando" la idea de un proyecto de acumulación de capital a partir del mejoramiento genético de plantas y venta de semillas (Ortoll, 2003). Asimismo, fue el trabajo diplomático de 
Rockefeller evidenciado en el entramado de alianzas, de aprendizaje en cooperación entre capitales externos y locales, más la ayuda invaluable de los gobiernos latinoamericanos, el que facilitó la consolidación de ese proyecto.

Como ya se mencionó, para la materialización de esta doble estrategia, tres importantes eventos focalizados en la investigación científica, la educación y la comunicación fueron fundamentales. En cuanto a la investigación y la educación, adicional a centros de investigación del germoplasma como el CIMMYT (primer evento), la creación en 1942 del Instituto Interamericano de Ciencias Agrícolas (IICA) (segundo evento) desempeñó un papel protagónico en relación con el desarrollo del servicio de extensión en América Latina durante la segunda mitad de la década de los cuarenta.

De manera conjunta con el Gobierno de los Estados Unidos, formó en su Escuela para Graduados (intercambios científicos) los primeros extensionistas, quienes posteriormente eran enviados a sus países con la idea de replicar los conocimientos o, en algunos casos, a integrarse a los ministerios de agricultura o instituciones relacionadas con el tema agrario, así como en la reorganización de los programas de ciencias agrarias en los países de América Latina. Desde allí se darían avances teóricos y experimentales en sociología rural, trascendiendo la esfera de lo estrictamente productivo, con el objetivo de aproximarse a la realidad rural comunitaria y pensar la forma más adecuada para potenciar los cambios sociales y culturales que era necesario generar sobre el campesino y las comunidades a fin de que este apropiara la idea del aumento de la productividad bajo el paradigma norteamericano.

Del lado de la comunicación (tercer evento), a finales de la década de los cincuenta, por iniciativa de la United Nations Educational, Scientific and Cultural Organization (Unesco), y con el respaldo de la Universidad Central del Ecuador, sería creado el Centro Internacional de Estudios Superiores de Comunicación para América Latina (Ciespal). La proyección de esta institución estaría fundamentada en la idea de la comunicación para el desarrollo, fruto de las recomendaciones de la Asamblea General de las Naciones Unidas, según la cual los países subdesarrollados deberían in- 
cluir en sus planes para el crecimiento económico la inversión de recursos para expandir y mejorar la comunicación, inspirados en estudios de investigadores como Daniel Lerner (1958), Everett Rogers (1962), Wilbur Schramm (1964) y académicos vinculados al Massachusetts Institute of Technology como Lucien Pye e Ithiel de Sola Poo. Se generó así todo un movimiento que, basado en los proyectos internacionales de alfabetización, extensión rural, radios comunitarias, servicios de salud, programas de crédito agrícola, entre otros, atribuía a los medios masivos de comunicación y a la educación un papel clave en los cambios sociales necesarios para la modernización (Sánchez, 1986).

Son varios los acontecimientos que pueden ser definidos como las raíces de la comunicación para el desarrollo. Sin embargo, todas tienen en común entender las estructuras cognoscitivas, culturales y conductuales en los países de la periferia como un obstáculo al desarrollo y, por tanto, ven en su transformación la posibilidad de cambiar sus modos de vida para hacerlos instrumentales a la reproducción del capital.

Así entonces, la comunicación para el desarrollo surge como un modelo de comunicación correspondiente con el modelo de desarrollo económico capitalista que los Estados Unidos buscaban difundir en aquel momento para legitimar el tipo de relaciones internacionales adecuado a sus intereses económicos, políticos y estratégicos. Para ello, contaba con una enorme superioridad científico-tecnológica que era la base de la doble estrategia mencionada de difusión científica y penetración cultural.

La primera escuela crítica latinoamericana de comunicación será formada, precisamente, por los jóvenes intelectuales reclutados para ese proyecto en los diferentes países de América Latina, para los cuales el enfoque funcionalista se presentará crecientemente como una barrera. Nuestra hipótesis de fondo es que, en esas condiciones, los aportes críticos del pensamiento histórico-estructural latinoamericano - que incluyen las teorías de la dependencia - se presentan como la opción natural que esos jóvenes investigadores encontrarán para avanzar en su rebeldía epistemológica. Con esto, se incorporan a todo un campo de pensamiento crítico que está en la base de lo que vendría a ser, más tarde, la EPC. 


\section{Hegemonía y subsunción por imposición de conocimiento}

Dos movimientos sucesivos marcan el proceso que lleva del precapitalismo, bajo el comando del capital mercantil europeo, a la Revolución Industrial y la implantación del modo de producción capitalista: uno de acumulación primitiva de conocimiento (Bolaño, 2013), que, en la producción mercantil, marca la transición del artesanado medieval a la industria, en el periodo manufacturero, y otro, de subsunción por imposición de conocimiento, en que el capital se vale de los desarrollos tecnológicos que pudo realizar según aquella expropiación originaria para revertir la relación sujeto-objeto e implantar su propio modo de producción.

En el mundo rural, este mismo proceso se presentará en un periodo mucho más amplio, que inicia en la constitución del mercado mundial, obra del gran capital mercantil y del absolutismo europeo, hasta la modernización del campo conocida como revolución verde en la segunda mitad del siglo XX. Entre uno y otro momento, procesos fundamentales de apropiación, deslegitimación e imposición de conocimiento se desarrollaron por todo el mundo; sin embargo, solo será a partir de la consolidación del proyecto modernizador de la potencia norteamericana al final de la Segunda Guerra Mundial que avanzarán las relaciones de producción específicamente capitalistas sobre el agro.

Al contrario de lo que ocurriera en los países del centro, en América Latina, el progreso tecnológico, incorporado inicialmente por la vía del consumo de las élites criollas, define un tipo de modernización que, favoreciendo inicialmente la incorporación imitativa de patrones de consumo y de vida, convirtió la dependencia cultural en un problema estructural del subdesarrollo. Es Celso Furtado quien aclara este problema, al incorporar el concepto antropológico de difusión cultural para entender la histórica dependencia cultural que América Latina ha vivido desde el momento mismo de la reconfiguración de su inserción en el mercado mundial durante la segunda mitad del siglo XIX, cuando los grupos internos consiguieron apropiarse parcialmente de la expansión del excedente, el cual tuvo como principal destino financiar una rápida diversificación de sus hábitos de consumo, vía importación de mercancías. Sin embargo, entrado el siglo XX, se hizo ne- 
cesario que parte de dicho excedente fuera canalizado hacia la compra de tecnología para acompañar el proceso de diversificación de patrones de consumo. Se dará durante este periodo, señala Furtado, el enraizamiento del problema de la dependencia cultural en el sistema de producción, es decir, la dependencia cultural ahora se torna también dependencia tecnológica.

En este sentido, es necesario ver en la difusión del progreso tecnológico centro-periferia un mecanismo que incide, no solo en la transformación de los modos de producción, sino también en el proceso de formación de la sociedad capitalista en la periferia, la cual requiere la generación de una serie de mediaciones para permitir la valorización del capital. Siguiendo el concepto de modo de vida propuesto por André Granou (1972), ${ }^{3}$ podemos plantear que, de la mano de la profundización del proceso de dependencia cultural, hay un proceso, principalmente a partir de la segunda mitad del siglo XX, de transformación de los modos de vida tradicionales en América Latina, entre ellos, el campesino.

Esos procesos son buen ejemplo de subsunción por imposición de conocimiento, pues, mientras son despojados los conocimientos tradicionales asociados a los cultivos y las prácticas pecuarias, así como el germoplasma de las variedades con que cuentan estos países, un nuevo conocimiento bajo el título de "científico" es difundido a técnicos, empresarios y productores. No queda duda, entonces, de que el capital intervino, y sigue interviniendo, de manera violenta, deslegitimando y subordinando las culturas tradicionales latinoamericanas en nombre de la ciencia occidental.

Es decir, mientras en el campo económico y político, involucrado en el proceso de industrialización por sustitución de importaciones se daba la expansión de los grandes conglomerados norteamericanos, de forma paralela en el campo social y cultural, involucrado en el proceso de revolución verde, se daba la imposición de las estructuras cognoscitivas, culturales y conductuales (valores y principios) ligados a la sociedad occidental. El

3 Por modo de vida debe entenderse "una totalidad concreta [citando a Kosik, 1963] producida y reproducida por el propio desarrollo del modo de producción, lo que exige analizar sus transformaciones como un proceso de destrucción del antiguo modo de vida, destrucción que es en sí misma una condición para que surja un nuevo modo de vida que coincida con las nuevas condiciones de producción” (Granou, 1972, p. 49). 
carácter modernizador de ambos procesos enmascaraba lo esencial, la dominación imperialista que era el garante de la implantación de las relaciones capitalistas en la periferia.

La doble estrategia aparece como mediadora de la difusión del progreso tecnológico a los países de la periferia, sometiendo de forma indirecta, no solo a un cercamiento en términos productivos a las comunidades rurales (al imponer al campesino la compra de todos los insumos necesarios para sus sistemas productivos y la reproducción de su familia, y la venta de su producción a estas grandes empresas), sino también en términos culturales, pues el conjunto de valores, principios y pautas de consumo asociadas a esas tecnologías modifican los modos de vida de las comunidades. Lo interesante de toda esta estrategia del capital es que, sin necesidad de un vínculo directo salarial ni la posesión de la tierra, como convencionalmente el capital lo hizo y lo sigue haciendo, recrea una novedosa forma de acumulación primitiva de capital. Así, podemos plantear que, vía subsunción por imposición de conocimiento, la modernización de la periferia ${ }^{4}$ conlleva, no solo la transformación de los modos de producción tradicionales, sino también, y principalmente, la transformación de los modos de vida.

En este proceso de modernización capitalista en América Latina, se evidencia una estrecha relación entre modelos de desarrollo, de ciencia, de educación y de comunicación, lo cual puede verse claramente en el caso que venimos exponiendo respecto de la difusión del progreso tecnológico que representó la revolución verde. En este caso, era fundamental transmitir a los campesinos la idea de que el aumento de la productividad era necesario para alcanzar el progreso y el desarrollo, que se verían reflejados en el incremento de la calidad de vida de las comunidades (es decir, la modernización). Es en este escenario que la comunicación para el desarrollo, basada en un modelo educativo que, en los términos de Paulo Freire, citado por Kaplún (1985), se denomina "modelo bancario" (de tipo funcionalis-

4 Furtado propone entender por esta idea "el proceso de adopción de patrones de consumo sofisticados (privados y públicos) sin el correspondiente proceso de acumulación de capital y progreso en los métodos de producción” (1975, p. 81). 
ta), sería utilizada para promover (imponer) un modelo de desarrollo de base capitalista, excluyente y deshumanizador.

Se habla respecto de la imposición de conocimiento, porque, lejos de ser una construcción conjunta de conocimiento para encontrar respuestas a los problemas específicos de las realidades latinoamericanas, este proceso se basa en la difusión de adaptaciones de tecnologías encontradas en los Estados Unidos a la realidad local soportadas en las innovaciones tecnológicas norteamericanas.

\section{Comunicación para el desarrollo: ¿un modelo para la deshumanización del desarrollo (comunicación dependiente)?}

\section{EI IICA y la formación de profesionales}

Remontándonos a la evolución histórica de las relaciones internacionales de los Estados Unidos con América Latina enunciadas, la creación del IICA se convierte en un hito fundador de la comunicación para el desarrollo, pues es el núcleo institucional que integra y replica muchos de los programas de educación, extensión rural, administración agropecuaria y de instrumentos teóricos y metodológicos que fundamentaron esas acciones. Uno de estos programas fue la formación de profesionales y posgraduados, con el cual un número importante de jóvenes latinoamericanos viajó a los Estados Unidos para formarse en diferentes áreas, con énfasis en lo agrícola. ${ }^{5}$ Tal es el caso de un grupo de jóvenes vinculados a proyectos de extensión y desarrollo agrícola que viajó en 1954 a Vermont (Burlington), para realizar visitas a centros y universidades con énfasis en desarrollo agrícola por invitación del Departamento de Agricultura de los Estados Unidos. Sería en esta experiencia que Juan Díaz Bordenave y Luis Ramiro Beltrán, dos grandes referentes de la escuela de pensamiento crítico latinoamericano en comunicación, se conocerían y establecerían una larga amistad en lo personal y lo académico. Fruto de ese ejercicio los dos llegarían posteriormente, en la segunda mitad

5 Conforme al IICA (1992), entre 1950 y 1959, fueron adiestrados 10000 profesionales en áreas como dasonomía, ecología, extensión agrícola, fitotecnia, entre otras. 
de la década de los sesenta, a la Universidad Estatal de Míchigan donde obtendrían el título de doctor en 1967 y 1968, respectivamente.

El papel protagónico que la agricultura (educación y extensión rural) aporta en el desarrollo de la comunicación como eje fundamental para la promoción del desarrollo en América Latina puede evidenciarse en el proceso laboral y de estudios que siguió Luis Ramiro Beltrán (tabla 1), el cual, tras iniciar en su natal Oruro el servicio de comunicador para la Secretaría de Sanidad, rápidamente viajaría a los Estados Unidos para recibir formación para convertirse en diseminador de esas ideas al retorno a su país. Posteriormente, ingresaría al IICA donde trabajaría durante dieciocho años. Allí participó del proceso de descentralización de esa institución (19501959), periodo en que se consolidaría el servicio de intercambio científico y el Proyecto 39 del Programa de Cooperación Técnica de la Organización de los Estados Americanos (OEA), así como del periodo denominado de nueva dimensión (1960-1969), momento en el que, bajo los fundamentos de la Alianza para el Progreso, la comunicación agrícola sería definida como una de las seis áreas de acción de esta institución (IICA, 1992).

\section{Tabla 1. Relación de la actividad laboral de Luis Ramiro Beltrán y las agencias de cooperación norteamericana (1954-1970)}

\begin{tabular}{|c|c|}
\hline Año & Actividad o trabajo asociado a los programas de cooperación norteamericana \\
\hline \multirow{2}{*}{1953} & Trabaja en la jefatura departamental de sanidad de Oruro adscrito al Servicio Agrícola Interamericano \\
\hline & $\begin{array}{l}\text { Participa del Primer Seminario Internacional de Educación Audiovisual auspiciado por la Foreign Operations Administration } \\
\text { (FOA) en Puerto Rico }\end{array}$ \\
\hline \multirow{2}{*}{1954} & $\begin{array}{l}\text { Viaja a los Estados Unidos, a Vermont, Burlington, por invitación del Departamento de Agricultura para realizar visitas a } \\
\text { centros y universidades con énfasis en desarrollo agrícola }\end{array}$ \\
\hline & $\begin{array}{l}\text { Hace escala de regreso en el IICA, Turrialba, Costa Rica, para familiarizarse con el Servicio de Intercambio Científico por } \\
\text { recomendación del estratega colombiano de desarrollo agrícola Armando Samper }\end{array}$ \\
\hline $1956-1957$ & Toma cursos de formación en comunicación para el desarrollo rural \\
\hline 1958 & $\begin{array}{l}\text { Indicado como especialista en información del Proyecto } 39 \text { del Programa de Cooperación Técnica de la Organización de } \\
\text { los Estados Americanos en Costa Rica en el IICA }\end{array}$ \\
\hline \multirow{3}{*}{1961} & Indicado como director del IICA Zona Andina \\
\hline & Dirige la misión de la Agencia para el Desarrollo Internacional de los Estados Unidos en Bolivia \\
\hline & $\begin{array}{l}\text { Primer Curso Nacional de Liderato en Comunicación Educativa en el Centro Audiovisual del Punto IV bajo la dirección } \\
\text { de Loren McIntyre }\end{array}$ \\
\hline $1965-1970$ & Maestría y doctorado en la Universidad del Estado de Míchigan bajo orientación de Everett Rogers y David Berlo \\
\hline 1971 & Director del Centro Internacional de Desarrollo Rural y Reforma Agraria (IICA-CIRA) \\
\hline
\end{tabular}

Fuente: elaboración propia según Beltrán (1971) y Herrera (2014). 


\section{El imperialismo cultural y la primera escuela crítica de comunicación en América Latina}

Ahora bien, en el trasegar de los pioneros en comunicación en América Latina (Beltrán, Bordenave, Pascuali, Kaplún, etc.), hay un punto de inflexión que definirá el surgimiento de la primera escuela crítica latinoamericana en comunicación. José Marques de Melo en su trabajo Los tiempos heroicos: la formación de la comunidad latinoamericana de ciencias de la comunicación (2004), documenta el periodo de 1972 a 1978 como el momento histórico que delimita la creación e institucionalización de Asociación Latinoamericana de Investigadores de la Comunicación (ALAIC), y lo llama la batalla por la hegemonía. Conforme al autor, ese periodo está marcado por una coyuntura particular en que se destaca la inclusión de la cuestión de la comunicación en el Movimiento de Países No Alineados, la incorporación de la investigación comunicacional en la agenda de las organizaciones intergubernamentales y la integración de América Latina en la geografía de la comunidad mundial de las ciencias de comunicación en el Congreso de la International Association for Media and Communication Research (IAMCR) en 1972.

Algunos hitos fundadores de este movimiento crítico en comunicación fueron, primero, la creación del Centro de Estudios de la Realidad Nacional (Ceren) en 1968 en la Universidad Católica de Chile, con la participación de Armand Mattelart y Héctor Schmucler, y posteriormente la creación de la revista Comunicación y Cultura de ese centro a inicios de la década de los setenta, propuestas teórico-críticas que bebían en aquel momento de la circulación de ideas sobre la dependencia en el laboratorio chileno (Beigel, 2006); y segundo, la invitación a Luis Ramiro Beltrán a la Conferencia Bienal de IAMCR en Leipzig (Alemania Oriental) en 1974, para hacer un balance crítico de la investigación comunicacional en la región, donde presentó el trabajo titulado Investigación comunicacional con los ojos vendados, en el cual "explicita la fértil trayectoria latinoamericana, finalizando con la duda sobre los caminos inmediatos, teniendo en vista el embate entre 'funcionalistas' y 'críticos'. Sugiere claramente una 'tercera vía', combinando el rigor metodológico de la tradición funcionalista con la osadía teórica de la corriente 'crítica”' (Marques de Melo, 2004, p. 14). 
Este contexto es bien definido por Aguirre (2016), quien, al referirse al trabajo de Bordenave y Beltrán, plantea que

\begin{abstract}
el gen del compromiso con la realidad social desde cada uno de sus puntos de origen fue significativo en cuanto sirvió para hablar en el seno mismo de la teorización de lo comunicativo a nombre de todo un continente, y justamente mostrar que en estas regiones la voluntad de comunicación y así de la mentada adopción de innovaciones no resultaba de la falta de voluntad hacia el cambio o hasta carencia de espíritu innovador de los campesinos, sean estos en Paraguay, Bolivia, Perú, Brasil, y otros lugares, sino que el modelo desarrollista de promoción de un cambio inducido por agencias de desarrollo, medios tecnológicos, más la presión de gobiernos, no se alcanzaba porque en medio estaba el factor de la desigualdad social, la mala distribución del poder en las estructuras políticas de las sociedades, y sobre todo hacía presencia la marcada pobreza de origen socio estructural e histórica en la que vivían grandes sectores de sus habitantes. (pp. 148-149)
\end{abstract}

Será, entonces, en torno a elementos como comunicación, cultura, subdesarrollo y dependencia que tendrán origen las teorías del imperialismo o de la dependencia cultural, la cual tendrá entre sus representantes, adicional a Matterlart y Schmucler, a Bordenave, Beltrán, Pasquali, Sunkel, Fuenzalida, Reyes Mata y Evelina Dagnino (Sarti, 1979). Para Sarti, la dependencia cultural representa un avance respecto de un par de teorías que criticaron profundamente las teorías de la comunicación y el desarrollo que colocaban a los medios de comunicación de masas como un instrumento capaz de atenuar el problema del subdesarrollo. ${ }^{6}$

Sin embargo, a pesar de superar los abordajes evolucionistas (etapas) y funcionalistas (modernización) que ven en el subdesarrollo una etapa atrasada del desarrollo, la solución, integrando al análisis de los medios de comunicación de masa el concepto althuseriano de aparatos ideológicos de Estado (AIE), los cuales, en América Latina, cumplían "la función ideológica de reforzar el carácter dependiente de las relaciones de producción" (Sarti, 1979, p. 235), sigue insuficiente, en la medida en que se

6 La primera vertiente se enfocó en la función ideológica de los medios masivos de comunicación, mientras la segunda se centró en el estudio sobre los contenidos de los mensajes. Ya la teoría de la dependencia estaría orientada al análisis de los efectos de esos procesos. 
hace hincapié en las relaciones entre naciones, dejando el análisis de clase en segundo plano.

Estando de acuerdo con la crítica de Sarti, Bolaño (2015) trata de señalar la necesidad de retomar la definición de dependencia cultural de Celso Furtado, anterior y distinta de las que informaron el campo de la comunicación en aquel momento. Aquí defendemos que una conexión entre la teoría estructuralista de la dependencia cultural de Furtado y la teoría marxista de la comunicación (Bolaño, 2013), no solo contribuiría a llenar el vacío señalado por Sarti respecto del análisis de clase en las teorías de la dependencia o del imperialismo cultural, sino que también sería clave para comprender el papel de los medios de comunicación en la mediación de la difusión del progreso tecnológico en América Latina que, en el campo, la visualizamos a través de la difusión de la revolución verde.

Desde el punto de vista epistemológico, se propone, en ese sentido, tender puentes - de forma no ecléctica en lo que nos concierne- entre los dos campos disciplinarios, la economía política y la comunicación, desde la génesis del pensamiento crítico latinoamericano, que la EPC hereda y trata de superar. Un ejemplo de articulación no ecléctica de ese tipo se presenta en lo que sigue.

\section{El concepto de efecto demostración y sus límites: la recepción crítica de Nurkse en Beltrán y Furtado y las bases teóricas de la EPC latinoamericana}

En 1971, Luis Ramiro Beltrán, como director del Centro Interamericano de Desarrollo Rural y Reforma Agraria (IICA-CIRA), publica un artículo titulado "La revolución verde y el desarrollo rural latinoamericano", en que da cuenta de la contradicción generada en el proceso de difusión de la revolución verde a América Latina. Advierte que, al contrario de lo esperado, los beneficios de la relación aumento de productividad-aumento de calidad de vida estaban quedando en manos de pocos, mientras el problema de la desigualdad social se estaba profundizando. 
En busca de respuestas a este problema, Beltrán trabaja un concepto con fuertes antecedentes en el estructuralismo latinoamericano: el efecto de demostración. ${ }^{7}$ Este concepto recuperado por Ragnar Nurkse a inicios de la década de los cincuenta para discutir el problema de la formación de capitales en los países subdesarrollados desde el lado de la demanda, coincide con los esfuerzos iniciales de Raúl Prebisch en la Comisión Económica para América Latina y el Caribe (Cepal), en el mismo periodo, por entender "la influencia ejercida sobre los países más pobres por los patrones de consumo de los más adelantados" (Furtado, 1997, t. 1, p. 260). La originalidad del trabajo de Nurkse radica en que introduce la propensión al consumo de los países subdesarrollados dentro de una teoría general del comportamiento del consumidor, al extrapolar la idea inicialmente planteada por James Duesenberry para el análisis de las desigualdades en la sociedad norteamericana al análisis de la desigualdad entre países (Furtado, 1952).

Conforme a la teoría del comportamiento del consumidor de Duesenberry, las funciones de consumo individuales son interdependientes (no independientes), de tal forma que el nivel de ahorro realizado por un individuo depende, no solo del nivel de su renta real, sino también, y posiblemente de manera predominante, de la relación entre su renta real y el nivel superior de la renta de otras personas con las cuales tiene contacto.

En esta perspectiva, Nurkse se pregunta si esta misma hipótesis puede ser válida para las relaciones económicas internacionales, es decir, si las funciones de consumo de los diferentes países son igualmente interdependientes. El autor advierte que, durante el proceso de difusión del progreso tecnológico a los países subdesarrollados, surgen nuevos productos "que modifican los modos de vida existentes y frecuentemente se tornan necesidades" (p. 53), planteando, entonces, que en esos países "es más fácil adoptar hábitos superiores de consumo que mejores métodos de producción”. Así entonces, un primer factor que intensifica el efecto de demostración es el tamaño de las disparidades de la renta real y de los patrones de consumo. Un

7 Conforme a Nurkse, este es un fenómeno según el cual, cuando los individuos establecen contacto con bienes superiores o patrones de consumo superiores, como nuevos artículos o nuevos medios para satisfacer viejas necesidades, quedan después de algún tiempo sujetos a cierta insatisfacción; su imaginación es estimulada, sus deseos y propensión al consumo son incrementados (1957, p. 50). 
segundo factor es el grado de conocimiento que el pueblo adquiere de tal fenómeno, y allí el autor otorga a la educación y a la comunicación un papel fundamental, pues "probablemente estos tienden a estimular los deseos antes que mejorar la productividad” (p. 56).

La recuperación que hace Beltrán del concepto está referida, principalmente, al segundo factor planteado por Nurkse: el grado de conocimiento del efecto, pues él lo entiende como una fuerza generadora de frustración, no en una perspectiva funcional, sino respecto de sus posibles consecuencias sobre la estabilidad de las relaciones sociales.

Para Beltrán, el problema es estructural:

\begin{abstract}
En la práctica, salvo muy contadas excepciones, la voluntad de cambio - la resolución política para procurar el verdadero desarrollo humano- parece todavía adormecida, si es que no ausente, de la escena latinoamericana. La minoría que domina la sociedad de esta región se resiste aún obstinadamente a ceder sus privilegios. La cuasi-colonial estructura de poder luce al principio de esta década no solo intacta en lo esencial, sino tal vez fortalecida en comparación al comienzo en la década anterior. (1971, p. 31)
\end{abstract}

En suma, al analizar la difusión de la revolución verde en América Latina, Beltrán define el efecto de demostración como un problema estructural asociado a la búsqueda del desarrollo, dejando de lado aquella hipótesis funcionalista conforme a la cual son los obstáculos cognitivos o "culturales" inherentes a estas comunidades que profundizan el subdesarrollo de las sociedades periféricas. En este sentido, se pregunta:

¿Cómo se puede esperar, en tales circunstancias, que haya un verdadero
desarrollo rural en las Américas? ¿Por qué se acusa a la masa campesina
de superstición y tradicionalismo, de ausencia de aptitud empresarial, de
indecisión de afrontar riesgos, de carencia de sentido del ahorro y del
afán de inversión, y de resistencia al cambio? ¿Es que no se percibe que
esas características negativas son el producto de la estructura social en
que esos campesinos viven atrapados? (1971, p. 18)

Beltrán adopta, de hecho, una idea ampliamente generalizada en el periodo, que entendía los medios de comunicación como directos respon- 
sables de promover e intensificar el efecto de demostración. Si bien para él es claro que bajo la revolución verde los medios de comunicación fueron claves para el proceso de difusión del progreso tecnológico, también es consciente de que estimularon procesos de concentración de la tierra y de los ingresos, en detrimento de las mayorías, de tal forma que la promesa del progreso técnico, el aumento de la productividad y la calidad de vida solo emergen como propulsores de frustración, de generación de promesas y sueños que nunca serán materializados. Deja claro, por tanto, que el problema se enraíza en la estructura social de las comunidades que actúa como propulsor de potenciales transformaciones en los modos de producción y especialmente en los modos de vida. ${ }^{8}$ Sin embargo, al intentar hacer una propuesta dentro del esquema democrático burgués, identifica la idea de voluntad política y la necesidad de planificación como ejes básicos para revertir la situación.

Así, por ejemplo, analizando el caso del Plan Puebla, ${ }^{9}$ proyecto que buscaba llevar los beneficios de la revolución verde a pequeños productores rurales en México, muestra cómo este incluso puede convertirse en una "revolución roja si su aplicación no va paralela con una dedicación política de justicia social que reduzca las grandes desigualdades económicas y sociales existentes en muchos de los países en vías de desarrollo" (Beltrán, 1971, p. 25).

En este aspecto, se encuentra perfectamente de acuerdo con el desarrollismo típico del momento histórico. Y es precisamente allí donde radica la limitante del enfoque, no solo de Beltrán, sino de la mayor parte de la escuela de pensamiento a que se filia. La perspectiva crítica de la EPC, como crítica interna a esa escuela, a partir de las décadas de los ochenta y noventa, tendrá justamente que superar esas limitaciones según las herramientas intelectivas del materialismo histórico.

8 Sobre las frustraciones, cita a Brown (1970): "Es probable que ellas sean una fuerza a favor del cambio mayor que ninguna tecnología o ideología introducidas jamás en los países pobres” (Beltrán, 1971, p. 11).

9 Sobre este proyecto ver, por ejemplo, Centro Internacional de Mejoramiento de Maíz y Trigo (s. f.). 
Las críticas de Celso Furtado al trabajo de Nurkse, por su parte, publicadas en la Revista Brasileira de Economia, de la Fundación Getúlio Vargas (FGV), en 1952, resaltan el rescate que hace este último de las limitantes a la formación de capital desde el lado de la demanda, en la medida en que la propensión al consumo resultante de las disparidades internacionales en la renta real emerge a la postre como un factor condicionante del desarrollo de los países de la periferia. Ahora bien, lo interesante de este planteamiento de Furtado es su reflexión respecto de las limitantes del análisis económico para entender el proceso histórico del desarrollo de la economía capitalista, pues para él la propensión al consumo es fuertemente influenciado por factores institucionales, lo cual está estrechamente relacionado con un amplio proceso de cambio cultural.

Ya hemos visto más arriba la perspectiva de Furtado sobre la relación entre cultura y desarrollo y su teoría de la dependencia cultural. Sin embargo, desde las críticas a Nurkse, a principios de la década de los cincuenta, el autor ya entiende que la dinámica del progreso tecnológico implica una secuencia lógica de generación de nuevos productos, modificación de modos de vida (patrón de vida) y generación de nuevas necesidades, de tal forma que, gracias a la enorme fuerza de los medios de propaganda y comunicación, los hábitos de consumo van en la frente, como el carro va adelante del burro (Furtado, 1952, p. 27). Posteriormente, Furtado (1955) explora la capacidad del concepto de excedente social para explicar los procesos de cambio social, en la medida en que hay una relación estrecha entre la organización social, la formación del excedente y la forma en que este es canalizado, pues solo ciertas formas de dominación social canalizan el excedente para la acumulación.

Son básicamente estos los elementos teóricos a partir de los cuales Furtado, durante el periodo de exilio, pasará a entender que el proceso de difusión del progreso tecnológico a América Latina se materializaría por la vía indirecta, es decir, resultado de la apropiación de los patrones de consumo y los modos de vida inherentes a las élites del centro, con una mínima penetración de las técnicas de producción responsables de la diversificación de este consumo. Sus análisis darían sustento, entonces, a una propuesta 
innovadora para entender el subdesarrollo latinoamericano, primero como un problema de dependencia cultural, y solo después, como un problema de dependencia tecnológica.

En este sentido, el fenómeno de dependencia tecnológica solo podría ocurrir cuando, fruto de la difusión del progreso tecnológico a inicios del siglo XX, se difundieron también patrones de consumo avanzados que solo podrían ser acompañados, si eran apropiadas técnicas de producción de igual nivel, de forma que la apropiación de tal tecnología tenía previamente enraizada la dependencia cultural inherente a las pautas de consumo, es decir, la difusión de la tecnología se daba como una necesidad, y única alternativa, para las élites locales de la periferia de acompañar los patrones de consumo del centro. En palabras de Furtado (1973), para entender lo que él denomina el desarrollo dependiente, la tecnología en la periferia, a diferencia de lo que sucedió con los países del centro, pasa de ser un medio para alcanzar el desarrollo, a ser un fin, pues, en estos últimos, el motor que dinamiza el subdesarrollo son las modificaciones en el perfil de la demanda y las respectivas modificaciones estructurales que se generan.

\section{Reconstruir caminos: estructuralismo latinoamericano y EPC}

Seguir esta línea argumentativa, partiendo de este viejo debate sobre el efecto demonstración, para llegar al problema del subdesarrollo, que, para Furtado, es prioritariamente, en el sentido en que lo estamos tratando de aclarar, un problema de dependencia cultural, nos sirve para recuperar la contribución de las teorías de la dependencia cultural, liberándolas de las limitantes bien señaladas por Sarti (1979).

Para ello, se hace necesario mostrar que la contradicción manifiesta del capital, que tradicionalmente se devela solo en la fórmula capital-trabajo (subsunción del trabajo en el capital), requiere de manera urgente evidenciar también, la contradicción economía/cultura (subsunción de la cultura popular en la economía). Es a partir del reconocimiento de esta doble contradicción desde donde la EPC brasileña hace sus aportes para la superación del debate sobre el efecto de demostración (Bolaño, 2015). 
Así entonces, reconociendo el papel clave de los medios de comunicación de masas en los problemas estructurales propios de las sociedades de Occidente, la EPC avanza en la discusión respecto del papel otorgado a los medios de comunicación de masas desde el imperialismo cultural, para situarlos en la discusión de las mediaciones sociales. Refiriéndose críticamente a los estudios culturales, Bolaño (2015) plantea el problema en los siguientes términos:

El problema es que al relegar a un segundo plano el problema de
orden económico que soporta la cultura de masas, jamás considera
el hecho, absolutamente esencial, de que la mediación es realiza-
da, de manera general, por capitales individuales en competencia,
que movilizan un tipo particular de trabajo (cultural o creativo, si se
prefiere) que es precisamente el elemento subjetivo que garantiza
efectivamente la colonización de la Lebenswelt, en los términos de
Habermas. (p. 101)

En este sentido, hay un proceso de apropiación por parte del capital de los elementos de la cultura popular que, pasando primero por un momento inicial de acumulación primitiva de conocimiento (Bolaño, 2013), constituirá posteriormente la cultura industrializada. En este sentido,

\begin{abstract}
la subsunción del trabajo cultural es lo que garantiza el diálogo permanente de la Industria Cultural con las culturas populares, dinamizándose el conjunto y garantizando, con eso, la reproducción de la hegemonía. Es a través del trabajo por tanto, que lo popular determina lo masivo y es a través del trabajo que el capital cultural ejerce su hegemonía sobre lo popular. La tecnología sirve para subsumir aquel trabajo popular particular, que dispone de conocimiento tácito derivado en su origen, de su extracción popular, de modo que para que la hegemonía se ejerza, la subsunción siempre será de alguna forma limitada siendo eso lo que explique las especificidades de la Economía Política de la Comunicación y la Cultura y no la herencia de la unicidad de la obra de arte como lo presenta la Economía Política de la Comunicación francesa. (Bolaño, 2015, p. 101)
\end{abstract}

En estos términos, entonces, la industria cultural emerge como la instancia de mediación característica del capitalismo monopolista, y es precisamente allí, apoyado en la teoría de la dependencia cultural de Furtado, desde donde la EPC brasileña supera la limitante ya identificada por Sar- 
tri (1979), al plantear que, bajo la idea de cultura de masas, se encubre la existencia de una cultura de clase. Así entonces,

\begin{abstract}
la industria cultural es la instancia de mediación característica del capitalismo monopolista, la forma específicamente capitalista de producción y difusión de bienes culturales, que se estructura a partir de la apropiación por el capital cultural (en el sentido de Marx, no de Bourdieu), de la cultura popular, a través del trabajo cultural que ella emplea. (Bolaño, 2015, p. 100)
\end{abstract}

Será esta la base teórica que la primera escuela crítica de la comunicación latinoamericana ignora y, a su vez, será la teoría que permita tejer un puente entre el estructuralismo latinoamericano y la EPC, para superar el concepto de efecto de demostración y encontrar en la industria cultural una nueva categoría que permita explicar la histórica dependencia cultural latinoamericana.

Si bien no cabe al objetivo de este trabajo avanzar en la aplicación de este referente teórico al problema abordado por Beltrán, sí consideramos importante, por lo menos, referir algunas ideas que esbozan el camino para emprender un análisis del proceso de integración al capitalismo del campesino latinoamericano durante el proceso de difusión de la revolución verde desde la década de los cincuenta. Proyectos como las estaciones experimentales norteamericanas durante la Segunda Guerra Mundial o las escuelas radiofónicas y los cuerpos de paz durante la segunda posguerra, cumplieron de manera particular este espacio de mediación, primero subvalorando y despojando a los campesinos de sus conocimientos tradicionales, su cultura tradicional, para posteriormente imponer un nuevo conocimiento (occidental) que transformaba los modos de vida tradicionales, colocando otros valores, principios y formas de ver y entender el mundo, ajenos a las realidades de las comunidades.

No nos es posible avanzar más en tal dirección en este trabajo, sin embargo, es importante resaltar que el retorno desde una perspectiva crítica a los principios de la primera escuela crítica latinoamericana de comunicación no se pretende realizar solo desde el plano teórico, al revés, por ejemplo, de la categoría de efecto de demostración, sino también en el plano de 
las acciones de la comunicación para el desarrollo, incluso aquellas paradigmáticas relacionadas con la comunicación rural. Queda, entonces, como una invitación para continuar el proyecto propuesto por la EPC desde la primera década del silgo XXI y que fue presentado de una manera más estructurada en el libro Campo aberto: para a crítica da epistemologia da comunicação (Bolaño, 2015).

\section{Referencias}

Aguirre, J. L. (2016). Diálogo entre entrañables comunicadores: Juan Díaz Bordenave y Luis Ramiro Beltrán Salmón. Revista Latinoamericana Ciencias de la Comunicación, 13(25), 144-154. Recuperado de http://www.alaic.org/revista/index.php/alaic/article/view/853

Beigel, F. (2006). Vida, muerte y resurrección de las teorías de la dependencia. En Autor, Crítica y teoría en el pensamiento social latinoamericano (pp. 287-326). Buenos Aires, Argentina: Consejo Latinoamericano de Ciencias Sociales.

Beltrán, L. R. (1971). La revolución verde y el desarrollo rural latinoamericano. Bogotá, Colombia: Centro Interamericano de Desarrollo Rural y Reforma Agraria.

Bolaño, C. (2013). Industria cultural, información y capitalismo. Barcelona, España: Gedisa.

Bolaño, C. (2015). Campo aberto: para a crítica da epistemologia da comunicação. Aracaju, Brasil: Editora do Diário Oficial de Sergipe.

Centro Internacional de Mejoramiento de Maíz y Trigo. (s. f.). Avances de un programa para aumentar rendimientos de maíz entre pequeños productores. Recuperado de https://libcatalog.cimmyt.org/Download/cim/26995.pdf

Furtado, C. (1952). Formação de capital e desenvolvimento econômico. Revista brasileira de Economia, 6(3), 7-45. Recuperado de http:// bibliotecadigital.fgv.br/ojs/index.php/rbe/article/view/2388 
Furtado, C. (1955). O desenvolvimento económico: ensaio de interpretação históricoanalitica. Econômica Brasileira, 1, 3-24.

Furtado, C. (1973). A hegemonia dos Estados Unidos e o subdesenvolvimento da América Latina. Río de Janeiro, Brasil: Civilização Brasileira.

Furtado, C. (1997). Obra autobiográfica (t. 1). Río de Janeiro, Brasil: Paz e Terra.

Granou, A. (1972). Capitalismo y modo de vida. Madrid, España: Alberto Corazón Editor.

Herrera Miller, K. M. (2004). ALAIC: el albergue de la inquietud. Entrevista al comunicólogo Luis Ramiro Beltrán. Revista Latinoamericana de Ciencias de la Comunicación, 1, 100-106.. DOI: 10.1109/ ICNDS.2010.5479203

Herrera Miller, K. M. (2014). El giro crítico: bases de la transformación del pensamiento comunicacional de Luis Ramiro Beltrán. Mediaciones, 10(12), 28-37. DOI: https://doi.org/10.26620/uniminuto.mediaciones.10.12.2014.28-37

Instituto Interamericano de Cooperación para la Agricultura. (1992). El IICA y su historia: 50 años de cooperación interamericana. San José, Costa Rica: Instituto Interamericano de Cooperación para la Agricultura.

Kaplún, M. (1985). El comunicador popular. Quito, Ecuador: Centro Internacional de Estudios Superiores de Comunicación para América Latina.

Marques de Melo, J. (2004). Los tiempos heroicos: la formación de la comunidad latinoamericana de ciencias de la comunicación. Recuperado de https://www.alaic.org/revista/index.php/alaic/article/viewFile/110/108 
Nurkse, R. (1957). Problemas da formação de capital em países subdesenvolvidos. Río de Janeiro, Brasil: Editôra Civilização Brasileira.

Ortoll, S. (2003). Orígenes de un proyecto agrícola: la Fundación Rockefeller y la revolución verde. Sociedades Rural, Producción y Media Ambiente, 4(1), 81-96. Recuperado de http://132.248.9.34/hevila/Sociedadesruralesproduccionymedioambiente/2003/vol4/ no1/8.pdf

Sánchez Ruiz, E. (1986). Réquiem por la modernización: perspectivas cambiantes en estudios del desarrollo. Ciudad de México, México: Universidad de Guadalajara.

Sarti, I. (1979). Comunicação e dependência cultural: um equivoco. En J. Werthein (Ed.), Meios de comunicação: realidade e mito (pp. 230251). São Paulo, Brasil: Nacional. 